\title{
Square Ising Nanowire on the Bethe Lattice
}

\author{
E. Albayrak* \\ Erciyes University, Department of Physics, 38039, Kayseri, Turkey
}

(Received March 9, 2016; in final form March 27, 2017)

\begin{abstract}
The square-type nanowire is simulated on the Bethe lattice by using the core-shell structure consisting of the Ising spins. A nanoparticle is formed by placing a spin to the center and four others to the corners of a square. Then, each nanoparticle is combined with two neighboring ones with a perfect alignment of the squares to form the nanowire. Only nearest-neighbor spin interactions, either ferromagnetic or antiferromagnetic type, are allowed. The phase diagrams are calculated by studying the thermal variations of magnetizations for various values of bilinear interactions. It is found that the model gives both second- and first-order phase transitions in addition to the tricritical points and compensation temperatures.
\end{abstract}

DOI: 10.12693/APhysPolA.131.1470

PACS/topics: Bethe lattice, nanowire, phase diagrams, magnetization, tricritical

\section{Introduction}

Lately, the study of magnetic materials at the size of nanoscales has been receiving more attention for experimental and theoretical reasons. These materials can be constructed by using different geometrical structures such as ultra-thin films, nanoparticles, nanowires, nanotubes, and so on. It is very important to study their magnetic behaviors because of their novel physical properties and possible applications in different areas of science and technology.

In this work, in order to construct a nanowire, first we built a nanoparticle by placing a spin to the center and four others to the corners of a square. Then, this nanoparticle is connected to similar nanoparticles from above and below and so on to form a square Ising nanowire. Nanowires of different geometries have been studied to investigate its different aspects such as; the magnetic properties [1], initial susceptibility in addition to the reduced total magnetization [2], hysteresis behaviors [3], critical and compensation temperatures [4], phase diagrams and magnetizations in the case of surface dilution [5], and dynamic behaviors [6]. They were all investigated by the use of effective-field theory (EFT) with correlations. The below works were also studied by the use of EFT with correlations for the spin- $1 / 2$ atoms: the effect of random magnetic field distribution on the phase diagrams and ground state magnetizations [7], magnetization, susceptibility and hysteresis loops described by the transverse Ising model [8], hysteresis behavior with core-shell morphology in a random magnetic field [9], phase diagrams of diluted Ising nanowire consisting of core and surface shell coupled by the exchange interaction [10] and cylindrical magnetic nanowire system composed of ferromagnetic core and shell layers in the presence of transverse fields and surface shell amorphization [11].

\footnotetext{
*e-mail: albayrak@erciyes.edu.tr
}

It should be mentioned that the triangular type nanowire on the Bethe lattice (BL) by using the core-shell structure consisting of the Ising spins were already examined in detail [12] where the thermal variations of magnetizations and the phase diagrams were obtained. In that work three surface spins, which were placed at the corners of a triangle, is extended to four surface spins in this work to form a square type of nanowire. Thus, the effect of increase of the number of surface spins may reveal some other characteristics of the system in comparison to [12] if there are any. This model also consists of a plaquette of spins, one at the center and four others on the surface, i.e. a nanoparticle, which is connected with other two plaquettes via the bilinear exchange interactions, then it is extended further to form the nanowire.

The formulation of the problem is obtained on the BL in terms of the exact recursion relations. As seen from the above literature, the nanowires were mostly studied by using the EFT. Therefore, the study of nanowires on the BL seems to be important since the BL is very useful in obtaining the exact results and it is very easy to adapt with different geometries. It is also useful to show that nanostructures can also be examined on the $\mathrm{BL}$ in terms of the exact recursion relations. The thermal variations of magnetizations are studied in both ferromagnetic $(\mathrm{F})$ and antiferromagnetic (AF) cases. Then, the phase diagrams are constructed by investigating the existence of possible phase transitions, i.e. second- or first-order, and critical points. The existence of compensation temperatures are also investigated.

The rest of this work is set up as follows. The formulation of problem is given in Sect. 2 on the BL in terms of the recursion relations. The phase diagrams are presented in the last section which includes some conclusions and comparisons.

\section{The formulation of square Ising nanowire on the Bethe lattice}

The Hamiltonian of the core-shell structured square nanowire can be given as 


$$
\mathcal{H}=-J_{c} \sum_{\langle i j\rangle} \sigma_{i} \sigma_{j}-J_{s} \sum_{\langle k l\rangle} S_{k} S_{l}-J_{s c} \sum_{\langle i k\rangle} \sigma_{i} S_{k},
$$

where $\sigma$ and $S$ spins are the Ising spins with the values $\pm 1 . J_{c}, J_{s}$ and $J_{s c}$ are the bilinear exchange interactions between the core spins, $\sigma$ 's, between the surface spins, i.e. the $S$ 's, and between the surface and the core spins, respectively. The model is given schematically in Fig. 1 where each plaquette represents a nanoparticle with one $\sigma$ spin and four $S$ spins. The index $i$ in $S_{i j}$ or $\sigma_{i}$ is the plaquette number and the index $j$ counts the spins on the surface of the plaquette. Only nearest-neighbor $(\mathrm{NN})$ spin interactions with appropriate $J$ values are allowed. It is also clear that each plaquette has only two NN nanoparticles, i.e. squares, thus $q=2$, the coordination number. First, we start with the partition

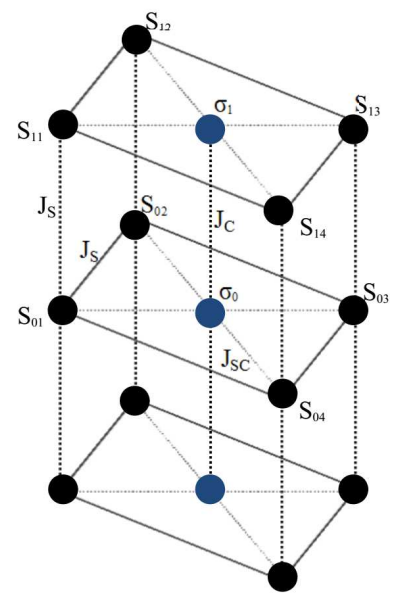

Fig. 1. Square Ising nanowire on the BL with coreshell structure consisting of Ising spins. $J_{c}, J_{s}$ and $J_{s c}$ are the bilinear exchange interactions between the core spins $\sigma$, between the surface spins $S$ and between the surface and the core spins, respectively. Each square represents a plaquette which are also connected via appropriate $J$ 's to form nanowire.

function given as

$$
Z=\sum_{\text {All Config }} \mathrm{e}^{-\beta \mathcal{H}}=\sum_{\{\sigma, S\}} P(\sigma, S),
$$

which has to be calculated by studying the probabilities depending on the spin values for the branches of the BL. Thus, if the BL is cut at the central plaquette, $i=0$, then

$$
\begin{aligned}
& P\left(\sigma_{0}, S_{01}, S_{02}, S_{03}, S_{04}\right)=\mathrm{e}^{\beta J_{s c} \sigma_{0}\left(S_{01}+S_{02}+S_{03}+S_{04}\right)} \\
\times & \mathrm{e}^{\beta J_{s}\left(S_{01} S_{02}+S_{02} S_{03}+S_{03} S_{04}+S_{04} S_{01}\right)} \\
\times & \prod_{j=1}^{q} Q_{n}\left[\left(\sigma_{0}, S_{01}, S_{02}, S_{03}, S_{04}\right) \mid\left(\sigma_{1}, S_{11}, S_{12}, S_{13}, S_{14}\right)^{j}\right],
\end{aligned}
$$

which gives the interactions between the central plaquette, or nanoparticle, spins as exponentials and the rest of the interactions are presented in the product. $q$ is the coordination number, the number of NN's for each of the nanoparticle. $j$ counts the number of the branches of the tree with the maximum of $q$ and $n$ counts the nanoparticles from root to the boundary sites. Now, if the BL is cut at the first plaquette, one gets

$$
Q_{n}\left[\left(\sigma_{0}, S_{01}, S_{02}, S_{03}, S_{04}\right) \mid\left(\sigma_{1}, S_{11}, S_{12}, S_{13}, S_{14}\right)^{j}\right]=
$$

$\mathrm{e}^{\beta J_{c} \sigma_{0} \sigma_{1}} \times \mathrm{e}^{\beta J_{s c} \sigma_{1}\left(S_{11}+S_{12}+S_{13}+S_{14}\right)}$

$\times \mathrm{e}^{\beta J_{s}\left(S_{11} S_{12}+S_{12} S_{13}+S_{13} S_{14}+S_{14} S_{11}\right)}$

$\times \mathrm{e}^{\beta J_{s}\left(S_{01} S_{11}+S_{02} S_{12}+S_{03} S_{13}+S_{04} S_{14}\right)}$

$\times \prod_{k=1}^{q-1} Q_{n-1}\left[\left(\sigma_{1}, S_{11}, S_{12}, S_{13}, S_{14}\right) \mid\left(\sigma_{2}, S_{21}, S_{22}, S_{23}, S_{24}\right)^{k}\right]$,

where the exponentials represent interactions in the first plaquette spins and between the central and first plaquette spins. The rest of the interactions are again included in the product term. Defining as:

$$
\begin{aligned}
& g_{n}\left(\sigma_{0}, S_{01}, S_{02}, S_{03}, S_{04}\right)= \\
& \sum_{\left\{\sigma_{0}, S_{01}, S_{02}, S_{03}, S_{04}\right\}} Q_{n}\left(\sigma_{0}, S_{01}, S_{02}, S_{03}, S_{04}\right),
\end{aligned}
$$

one obtains the partition function in the form of

$$
\begin{aligned}
Z & =\sum_{\left\{\sigma_{0}, S_{01}, S_{02}, S_{03}, S_{04}\right\}} \mathrm{e}^{\beta J_{s c} \sigma_{0}\left(S_{01}+S_{02}+S_{03}+S_{04}\right)} \\
& \times \mathrm{e}^{\beta J_{s}\left(S_{01} S_{02}+S_{02} S_{03}+S_{03} S_{04}+S_{04} S_{01}\right)} \\
& \times\left[g_{n}\left(\sigma_{0}, S_{01}, S_{02}, S_{03}, S_{04}\right)\right]^{q}
\end{aligned}
$$

Now from Eqs. (4) and (5), one can easily obtain

$$
\begin{aligned}
& g_{n}\left(\sigma_{0}, S_{01}, S_{02}, S_{03}, S_{04}\right)=\sum_{\left\{\sigma_{1}, S_{11}, S_{12}, S_{13}, S_{14}\right\}} \\
& \times \mathrm{e}^{\beta J_{c} \sigma_{0} \sigma_{1}} \mathrm{e}^{\beta J_{s c} \sigma_{1}\left(S_{11}+S_{12}+S_{13}+S_{14}\right)} \\
& \times \mathrm{e}^{\beta J_{s}\left(S_{11} S_{12}+S_{12} S_{13}+S_{13} S_{14}+S_{14} S_{11}\right)} \\
& \times \mathrm{e}^{\beta J_{s}\left(S_{01} S_{11}+S_{02} S_{12}+S_{03} S_{13}+S_{04} S_{14}\right)} \\
& \quad \times\left[g_{n-1}\left(\sigma_{1}, S_{11}, S_{12}, S_{13}, S_{14}\right)\right]^{q-1} .
\end{aligned}
$$

These partial partition functions, i.e. $g_{n}(\sigma, S)$ 's, depend on the different spin configurations of the model and its number is calculated from (number of spin values) number of spins, $^{\text {i.e. }} 2^{5}=32$ in our case. The exact recursion relations are obtained by taking the ratios of the $g_{n}(\sigma, S)$ 's for each of the separate branches of the BL. Note that the recursion relations serve the role of the equations of states. The possible spin configurations and the $2^{5}-1=31$ recursion relations are given below by considering a nanoparticle, i.e. the square, with one core and four surface spins as

$$
\begin{array}{ll}
X_{1}=\frac{g_{n}(+++++)}{g_{n}(-----)}, & X_{2}=\frac{g_{n}(++++-)}{g_{n}(-----)}, \\
X_{3}=\frac{g_{n}(+++-+)}{g_{n}(-----)}, & X_{4}=\frac{g_{n}(++-++)}{g_{n}(-----)}, \\
X_{5}=\frac{g_{n}(+-+++)}{g_{n}(-----)}, & X_{6}=\frac{g_{n}(-++++)}{g_{n}(-----)},
\end{array}
$$




$$
\begin{aligned}
& X_{7}=\frac{g_{n}(+++--)}{g_{n}(-----)}, \quad X_{8}=\frac{g_{n}(++-+-)}{g_{n}(-----)}, \\
& X_{9}=\frac{g_{n}(+-++-)}{g_{n}(-----)}, \quad X_{10}=\frac{g_{n}(-+++-)}{g_{n}(-----)}, \\
& X_{11}=\frac{g_{n}(++--+)}{g_{n}(-----)}, \quad X_{12}=\frac{g_{n}(+-+-+)}{g_{n}(-----)}, \\
& X_{13}=\frac{g_{n}(-++-+)}{g_{n}(-----)}, \quad X_{14}=\frac{g_{n}(+--++)}{g_{n}(-----)}, \\
& X_{15}=\frac{g_{n}(-+-++)}{g_{n}(-----)}, \quad X_{16}=\frac{g_{n}(--+++)}{g_{n}(-----)}, \\
& X_{17}=\frac{g_{n}(++---)}{g_{n}(-----)}, \quad X_{18}=\frac{g_{n}(+-+--)}{g_{n}(-----)}, \\
& X_{19}=\frac{g_{n}(+--+-)}{g_{n}(-----)}, \quad X_{20}=\frac{g_{n}(+---+)}{g_{n}(-----)}, \\
& X_{21}=\frac{g_{n}(-++--)}{g_{n}(-----)}, \quad X_{22}=\frac{g_{n}(-+-+-)}{g_{n}(-----)}, \\
& X_{23}=\frac{g_{n}(-+--+)}{g_{n}(-----)}, \quad X_{24}=\frac{g_{n}(--++-)}{g_{n}(-----)}, \\
& X_{25}=\frac{g_{n}(--+-+)}{g_{n}(-----)}, \quad X_{26}=\frac{g_{n}(---++)}{g_{n}(-----)}, \\
& X_{27}=\frac{g_{n}(+----)}{g_{n}(-----)}, \quad X_{28}=\frac{g_{n}(-+---)}{g_{n}(-----)}, \\
& X_{29}=\frac{g_{n}(--+--)}{g_{n}(-----)}, \quad X_{30}=\frac{g_{n}(---+-)}{g_{n}(-----)}, \\
& X_{31}=\frac{g_{n}(----+)}{g_{n}(-----)},
\end{aligned}
$$

which are too long to be given explicitly in this work. After having calculated the recursion relations, we are now ready to obtain the partition function and magnetizations in terms of them. Thus, the partition function is given as

$$
\begin{aligned}
Z & =\mathrm{e}^{4 \beta^{\prime}(p+r)} X_{1}^{q}+\mathrm{e}^{2 \beta^{\prime} r} X_{2}^{q}+\mathrm{e}^{2 \beta^{\prime} r} X_{3}^{q}+\mathrm{e}^{2 \beta^{\prime} r} X_{4}^{q} \\
& +\mathrm{e}^{2 \beta^{\prime} r} X_{5}^{q}+\mathrm{e}^{4 \beta^{\prime}(p-r)} X_{6}^{q}+X_{7}^{q}+\mathrm{e}^{-4 \beta^{\prime} p} X_{8}^{q}+X_{9}^{q} \\
& +\mathrm{e}^{-2 \beta^{\prime} r} X_{10}^{q}+X_{11}^{q}+\mathrm{e}^{-4 \beta^{\prime} p} X_{12}^{q}+\mathrm{e}^{-2 \beta^{\prime} r} X_{13}^{q}+X_{14}^{q} \\
& +\mathrm{e}^{-2 \beta^{\prime} r} X_{15}^{q}+\mathrm{e}^{-2 \beta^{\prime} r} X_{16}^{q}+\mathrm{e}^{-2 \beta^{\prime} r} X_{17}^{q}+\mathrm{e}^{-2 \beta^{\prime} r} X_{18}^{q} \\
& +\mathrm{e}^{-2 \beta^{\prime} r} X_{19}^{q}+\mathrm{e}^{-2 \beta^{\prime} r} X_{20}^{q}+X_{21}^{q} \\
& +\mathrm{e}^{-4 \beta^{\prime} p} X_{22}^{q}+X_{23}^{q}+X_{24}^{q}+\mathrm{e}^{-4 \beta^{\prime} p} X_{25}^{q}+X_{26}^{q} \\
& +\mathrm{e}^{4 \beta^{\prime}(p-r)} X_{27}^{q}+\mathrm{e}^{2 \beta^{\prime} r} X_{28}^{q}+\mathrm{e}^{2 \beta^{\prime} r} X_{29}^{q} \\
& +\mathrm{e}^{2 \beta^{\prime} r} X_{30}^{q}+\mathrm{e}^{2 \beta^{\prime} r} X_{31}^{q}+\mathrm{e}^{4 \beta^{\prime}(p+r)} .
\end{aligned}
$$

The magnetization of the core spin, $\sigma_{0}$, of the central nanoparticle, or the square, is calculated as

$$
\begin{aligned}
& M_{c}=\left\langle\sigma_{0}\right\rangle=\left[\mathrm{e}^{4 \beta^{\prime}(p+r)} X_{1}^{q}+\mathrm{e}^{2 \beta^{\prime} r} X_{2}^{q}+\mathrm{e}^{2 \beta^{\prime} r} X_{3}^{q}\right. \\
& +\mathrm{e}^{2 \beta^{\prime} r} X_{4}^{q}+\mathrm{e}^{2 \beta^{\prime} r} X_{5}^{q}-\mathrm{e}^{4 \beta^{\prime}(p-r)} X_{6}^{q}+X_{7}^{q} \\
& +\mathrm{e}^{-4 \beta^{\prime} r} X_{8}^{q}+X_{9}^{q}-\mathrm{e}^{-2 \beta^{\prime} r} X_{10}^{q}+X_{11}^{q} \\
& +\mathrm{e}^{-4 \beta^{\prime} r} X_{12}^{q}-\mathrm{e}^{-2 \beta^{\prime} r} X_{13}^{q}+X_{14}^{q}-\mathrm{e}^{-2 \beta^{\prime} r} X_{15}^{q} \\
& -\mathrm{e}^{-2 \beta^{\prime} r} X_{16}^{q}+\mathrm{e}^{-2 \beta^{\prime} r} X_{17}^{q}+\mathrm{e}^{-2 \beta^{\prime} r} X_{18}^{q}+\mathrm{e}^{-2 \beta^{\prime} r} X_{19}^{q}
\end{aligned}
$$

$$
\begin{aligned}
& +\mathrm{e}^{-2 \beta^{\prime} r} X_{20}^{q}-X_{21}^{q}-\mathrm{e}^{-4 \beta^{\prime} p} X_{22}^{q}-X_{23}^{q}-X_{24}^{q} \\
& -\mathrm{e}^{-4 \beta^{\prime} p} X_{25}^{q}-X_{26}^{q}+\mathrm{e}^{4 \beta^{\prime}(p-r)} X_{27}^{q}-\mathrm{e}^{2 \beta^{\prime} r} X_{28}^{q} \\
& \left.-\mathrm{e}^{2 \beta^{\prime} r} X_{29}^{q}-\mathrm{e}^{2 \beta^{\prime} r} X_{30}^{q}-\mathrm{e}^{2 \beta^{\prime} r} X_{31}^{q}-\mathrm{e}^{4 \beta^{\prime}(p+r)}\right] / Z
\end{aligned}
$$

and the magnetization of one of the surface spins which are all equivalent is obtained, for example, for $S_{01}$ as

$$
\begin{aligned}
& M_{s}=\left\langle S_{01}\right\rangle=\left[\mathrm{e}^{4 \beta^{\prime}(p+r)} X_{1}^{q}+\mathrm{e}^{2 \beta^{\prime} r} X_{2}^{q}+\mathrm{e}^{2 \beta^{\prime} r} X_{3}^{q}\right. \\
& +\mathrm{e}^{2 \beta^{\prime} r} X_{4}^{q}-\mathrm{e}^{2 \beta^{\prime} r} X_{5}^{q}+\mathrm{e}^{4 \beta^{\prime}(p-r)} X_{6}^{q}+X_{7}^{q} \\
& +\mathrm{e}^{-4 \beta^{\prime} p} X_{8}^{q}-X_{9}^{q}+\mathrm{e}^{-2 \beta^{\prime} r} X_{10}^{q}+X_{11}^{q} \\
& -\mathrm{e}^{-4 \beta^{\prime} p} X_{12}^{q}+\mathrm{e}^{-2 \beta^{\prime} r} X_{13}^{q}-X_{14}^{q}+\mathrm{e}^{-2 \beta^{\prime} r} X_{15}^{q} \\
& -\mathrm{e}^{-2 \beta^{\prime} r} X_{16}^{q}-\mathrm{e}^{-2 \beta^{\prime} r} X_{17}^{q}-\mathrm{e}^{-2 \beta^{\prime} r} X_{18}^{q}-\mathrm{e}^{-2 \beta^{\prime} r} X_{19}^{q} \\
& -\mathrm{e}^{-2 \beta^{\prime} r} X_{20}^{q}+X_{21}^{q}+\mathrm{e}^{-4 \beta^{\prime} p} X_{22}^{q}+X_{23}^{q}-X_{24}^{q} \\
& -\mathrm{e}^{-4 \beta^{\prime} p} X_{25}^{q}-X_{26}^{q}-\mathrm{e}^{4 \beta^{\prime}(p-r)} X_{27}^{q}+\mathrm{e}^{2 \beta^{\prime} r} X_{28}^{q} \\
& \left.-\mathrm{e}^{2 \beta^{\prime} r} X_{29}^{q}-\mathrm{e}^{2 \beta^{\prime} r} X_{30}^{q}-\mathrm{e}^{2 \beta^{\prime} r} X_{31}^{q}-\mathrm{e}^{4 \beta^{\prime}(p+r)}\right] / Z(11)
\end{aligned}
$$

where $p=J_{s} / J_{c}, r=J_{s c} / J_{c}$ and $\beta^{\prime}=\beta J_{c}=J_{c} / k T$. Thus the magnetization of the central plaquette, i.e. square, can be calculated from

$$
M_{T}=\left(M_{c}+4.0 M_{s}\right) / 5.0 \text {. }
$$

These are all the necessary formulations for studying the core-shell structured square nanowire in terms of the exact recursion relations on the BL. In the next section, we study the thermal variations of magnetizations to obtain the phase diagrams on the possible planes of our model.

\section{The phase diagrams of the square nanowire, results and conclusions}

In this section, the phase diagrams are illustrated on the $\left(p, k T / J_{c}\right)$ and $\left(r, k T / J_{c}\right)$ planes for given values of $r$ and $p$, respectively, by studying the thermal variations of magnetizations of the central plaquette. The bilinear interaction parameter $J_{c}$ between the core spins is taken as a scaling factor, or equally set equal to 1.0, which means that the interactions between core spins are $\mathrm{F}$ type. The signs of $J_{s}$ or $p$ and $J_{s c}$ or $r$, respectively, control whether the interactions between surface spins and between core and surface spins are $\mathrm{F}$ or AF type. According to our Hamiltonian, when $J>0$, the interactions are of $\mathrm{F}$ type and when $J<0$, they are of AF type. In the phase diagrams, the solid- and dashed lines, i.e. $T_{c^{-}}$ and $T_{t}$-lines, represent the lines of second- and first-order phase transitions, respectively. The phase is paramagnetic $(\mathrm{P})$ above any of these lines. The solid triangles indicate tricritical points at which $T_{c^{-}}$and $T_{t}$-lines meet. The phase diagram on the $\left(r, k T / J_{c}\right)$ plane is obtained for given values of $p$ and illustrated in Fig. 2. The lines are labeled with the values of $p>0.0$, which only leads to $\mathrm{F}$ type of interactions. As for $r$, the interactions are either F or AF type for the right- and left-sides of the plane, respectively. For higher $p$ values, the wing-shaped $T_{c}$-lines are quite symmetric with respect to the origin 
of the plane. But this symmetry is spoiled at lower $p$ 's about zero $r$. These lines reach minimum temperatures at zero $r$ for higher $p$ values. Two minima are seen for $p=0.1$ and 0.05 about zero $r$. Again one minimum is seen to the left of $r=0.0$ for $p=0.01$ and 0.0 . This figure is qualitatively similar with Fig. 3a of [12], but here the $T_{c}$-lines are seen at little higher temperatures. This is a well-known behavior of the BL, that is, as the coordination number increases the critical temperatures are seen at higher temperatures. Even if in both works $q$ equals 2 , the number of the surface spins is increased in here by one which leads to the same effect as expected.

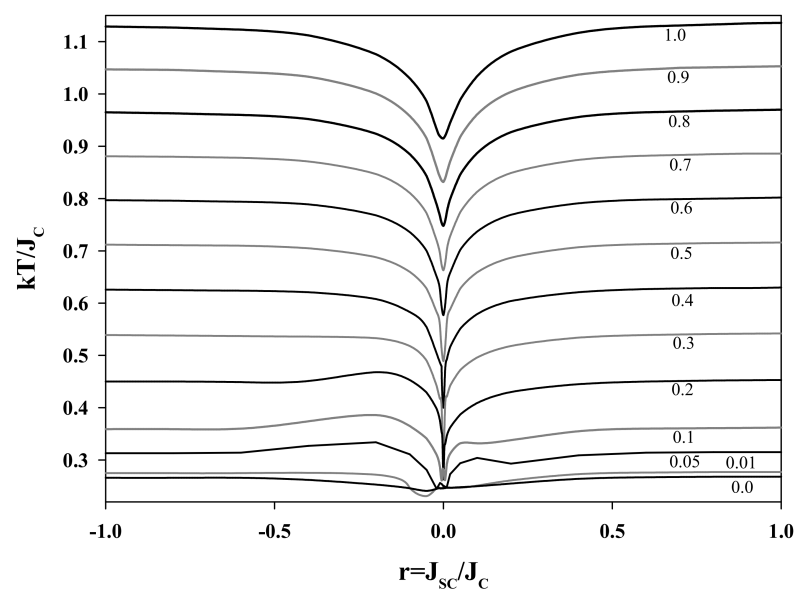

Fig. 2. The phase diagram of the model on the $\left(r, k T_{c} / J\right)$ plane for given values of $p$.

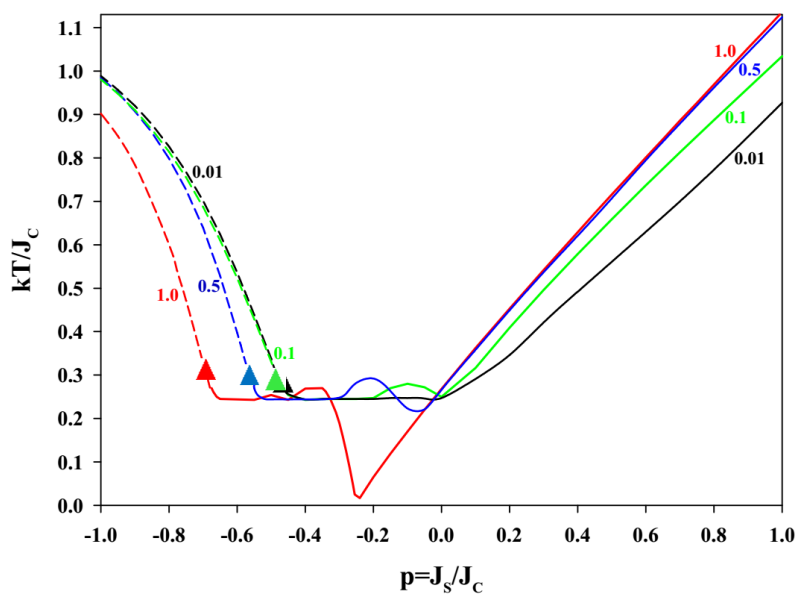

Fig. 3. The phase diagram of the model on the $\left(p, k T_{c} / J\right)$ plane for given values of $r$.

The last phase diagram, Fig. 3, is obtained on the $\left(p, k T / J_{c}\right)$ plane for positive $r$ values. Therefore, when $p$ is also positive, all the interactions are of $\mathrm{F}$ type as seen from the right-hand side of the figure. It is well-known that as $J$ values increase positively, the spins bond together strongly ferromagnetically, therefore, higher temperatures are required to drive the system to paramagnetic case. This is clear from the figure that temperatures of these lines increase as $p$ and $r$ increase. In going from $r>0$ to the $r<0$ case, first we see some wiggling in the $T_{c}$-lines which may be caused by the existence of some frustrations which was also the case in [12]. As $p$ becomes more negative, each $T_{c}$-lines terminate at tricritical points from where the $T_{t}$-lines appear. The tricritical points are seen at lower $p$ 's for higher $r$ 's and at little higher temperatures. The existence of the $T_{t}$-lines and tricritical points are different than [12], this may be caused by adding one more spins to the surface of each nanoparticles which has increased the temperatures of these critical lines. Note also that the $T_{t}$-lines increase in temperature as $p$ increases negatively.

In conclusion, the Ising nanowire formed from the square type of nanoparticles on the BL by using the coreshell structure consisting of the Ising spins. Each of plaquettes consists of one spin at the center and four other spins on the surface which is then connected with the other two plaquettes via bilinear exchange interactions. The formulation of the problem on the BL is obtained in terms of the exact recursion relations. The thermal variations of magnetizations are studied for both $\mathrm{F}$ and AF cases to obtain the phase diagrams. Increase of the number of the surface spins has changed phase diagrams as explained above. We have also found the existence of compensation temperatures but we did not go into any details since they are similar with [12]. It should be noted that adding one spin to the surface of each nanoparticle almost doubles the number of recursion relations on the BL, i.e. from 15 to 31 . It should also be mentioned that the square Ising nanowire is constructed from the combinations of the Ising nanoparticles, so when $r=0$, it does not decouple into an Ising chain consisting of $\sigma$ spins and a square surface shell of $s$-spins. Therefore, the model presented in here must be considered as a whole.

\section{References}

[1] T. Kaneyoshi, Phys. Status Solidi B 248, 250 (2011).

[2] T. Kaneyoshi, J. Magn. Magn. Mater. 323, 2483 (2011).

[3] M. Keskin, N. Sarli, B. Deviren, Solid State Commun. 151, 1025 (2011).

[4] T. Kaneyoshi, Physica A 390, 3697 (2011).

[5] T. Kaneyoshi, Physica A 391, 3616 (2012).

[6] M. Ertaş, Y. Kocakaplan, Phys. Lett. A 378, 845 (2014).

[7] Ü. Akinci, J. Magn. Magn. Mater. 324, 3951 (2012).

[8] S. Bouhou, I. Essaoudi, A. Ainane, M. Saber, F. Dujardin, J.J. de Miguel, J. Magn. Magn. Mater. 324, 2434 (2012).

[9] A. Zaim, M. Kerouad, M. Boughrara, J. Magn. Magn. Mater. 331, 37 (2013).

[10] S. Bouhou, I. Essaoudi, A. Ainane, M. Saber, R. Ahuja, F. Dujardin, J. Magn. Magn. Mater. 336, 75 (2013).

[11] Y. Yüksel, Ü. Akinci, H. Polat, Physica A 392, 2347 (2013).

[12] E. Albayrak, Phys. Lett. A 380, 458 (2016). 